\title{
Cortical lateralization in stroke patients measured by event-related potentials during motor imagery
}

\author{
WEIJUN GONG, TONG ZHANG and LEI SHAN \\ Department of Neurological Rehabilitation, Beijing Boai Hospital, China Rehabilitation Research Center, \\ Capital Medical University, Beijing 100068, P.R. China
}

Received April 16, 2013; Accepted August 28, 2013

DOI: $10.3892 / \mathrm{mmr} .2013 .1705$

\begin{abstract}
Stroke is a leading cause of impairment and disability worldwide, and motor imagery (MI) has been used in stroke rehabilitation. Electroencephalography (EEG) has been used to study MI. However, the characteristic features of EEG during MI in stroke patients have not been established. The purpose of this study was to investigate the difference in event-related potentials (ERPs) during MI between healthy controls and stroke patients. This study included nine stroke patients and nine healthy age-matched controls, who performed tasks involving MI, passive movement without MI and passive movement with MI. One hundred and twenty-eight channel ERPs were recorded to capture cerebral activation. Electrodes E44 and E120 (corresponding to the inferior precentral area) were selected to analyze the lateralization effects of ERPs. Lateralization was calculated as the ratio of the potential at $500 \mathrm{~ms}$ at electrode E120 to that at electrode E44. In the controls, the different ERPs exhibited differential direction between the 0-300 and the 300-700 ms interval. ERPs were evoked by passive movement with MI and MI alone, but not passive movement without MI. In addition, a lateralization effect in control patients as shown by the observation that the lateralization ratio in passive movement with MI and MI alone was significantly different from that in passive movement without MI $(\mathrm{P}<0.05)$. The amplitudes of the different ERPs were significantly smaller in stroke patients compared with those in the controls $(\mathrm{P}<0.05)$. The lateralization ratio in the stroke patients was opposite and significantly different from that of the controls $(\mathrm{P}<0.05)$. The results suggested that the MI-induced lateralization effect in ERPs may be used as a measure for evaluating the MI impairment and recovery in stroke patients.
\end{abstract}

Correspondence to: Dr Tong Zhang, Department of Neurological Rehabilitation, Beijing Boai Hospital, China Rehabilitation Research Center, Capital Medical University, 10 Jiaomen North Road, Beijing 100068, P.R. China

E-mail: tongzhang1290@gmail.com

Key words: event-related potentials, motor imagery, stroke, rehabilitation

\section{Introduction}

Stroke is a leading cause of impairment and disability worldwide. The most common impairment resulting from stroke is motor impairment, which typically affects the control of movement on one side of the body. Rehabilitation strategies for stroke patients have primarily focused on the recovery of impaired movement and its associated motor function (1). Motor imagery (MI) practice has been used in stroke rehabilitation $(2,3)$ and has produced positive effects in hand movement, sit-to stand performance and activities of daily living (4-6).

MI has been defined as the mental representation of movement without any body movement $(7,8)$. It is a complex cognitive process that is involved in the reactivation of specific motor actions within working memory $(9,10)$. Neuroimaging studies have shown that MI activates more or less the same brain regions as the actual execution of a movement, including motor and premotor areas, the prefrontal cortex and the parietal cortex (10-15). Studies have demonstrated the benefits of MI in improving motor performance in patients with neurological diseases (16).

Electroencephalography (EEG), a noninvasive and convenient method to record brain signals, has been used to study MI. MI desynchronizes the ongoing EEG activity and this event-related phenomena may be due to desynchronized activities of the activated neurons during the processing of cognitive information or the production of motor behavior, termed event-related desynchronization (ERD) (17). MI is associated with ERD in EEG over motor cortical areas, particularly at the contralateral hemisphere (18). ERD has been used to analyze the brain activity in stroke patients (19-21). However, it has been observed that ERD patterns are similar during MI, motor execution and passive movement $(18,22,23)$. Failure to detect MI through ERD patterns prevents the effective evaluation of MI execution during training and functional recovery following MI training. Therefore, identification of an EEG measure to distinguish between MI and passive movement will aid in elucidating an effective method to analyze MI in stroke patients.

In the present study, the event-related potentials (ERPs) measured with EEG from stoke patients and healthy controls performing tasks involving MI, passive movement without MI and passive movement with MI tasks were investigated. The 
purpose of this study was to identify the differences in ERPs for MI and passive movement in stroke patients and controls.

\section{Subjects and methods}

Subjects. This study was approved by the Research Ethics Committee of Beijing Boai Hospital (Beijing, China) and all subjects provided their informed consent. Nine stroke patients (5 males and 4 females) were selected from the Neurology Department of the Capital Medical University (Beijing, China). Patients were selected according to the following criteria: i) Male or female patients whose age ranged from 25 to 45 years; ii) cerebral infarction or hemorrhage was diagnosed according to the report from the the classification of cerebrovascular diseases III of the National Institute of Neurological Disorders and Stroke (24); iii) cerebral infarction or hemorrhage in the basal ganglion confirmed by brain computerized tomography (CT) or magnetic resonance imaging (MRI); iv) disease duration of 3-6 months; and v) no history of other neurological diseases. Patients with the following conditions were excluded: i) patients with lesions in the cerebellum and cortex observed by MRI or CT; ii) impaired cognitive function with the Mini-Mental State Examination (score of <17); iii) patients who were unable or unwilling to complete the study and; iv) patients with severe diseases of the heart, liver, kidney, brain or hematopoietic system, as well as epilepsy, infectious diseases or brain trauma. Only patients with subcortical stroke were selected in order to exclude the possible effects of lesions in the cerebellum and cortex on MI.

Nine age-matched healthy volunteers $(5$ males and 4 females) were selected according to the following criteria: i) male or female patients whose age ranged from 25 to 45 years; ii) no history of neurological or psychiatric disease; iii) normal brain CT and MRI; iv) no history of trauma or administration of medicine within 3 months prior to the study; v) no severe diseases that affected the performance of the experiments; and vi) willingly cooperated with the examination.

Tasks. Subjects performed the following tasks: MI, passive movement with no MI and passive movement with MI. The subjects were seated in front of a 17-inch screen located at a distance of $1 \mathrm{~m}$. The subject's hand and forearm were held still and the index fingers were not in contact with any objects. Only index fingers were moved by the examiner for passive movement tasks and all the other fingers rested on the table. Subjects were requested not to move their eyes prior to and during tasks, but to look at a fixation cross on the screen. For each subject, the left and right index fingers were tested. For each finger, the tasks consisted of 12 sessions, with 4 sessions composed of 20 trials for each experimental condition (MI, passive movement with MI and passive movement without MI). There were a total of 80 trials per condition for each finger. Sections were randomized and started with a $10 \mathrm{sec}$ presentation of a word sign on the screen to instruct the subjects to prepare for the MI, passive movement with no MI or passive movement with MI. For MI tasks, subjects were asked to imagine the movements of the finger (flexion or extension of the index finger) without actually performing

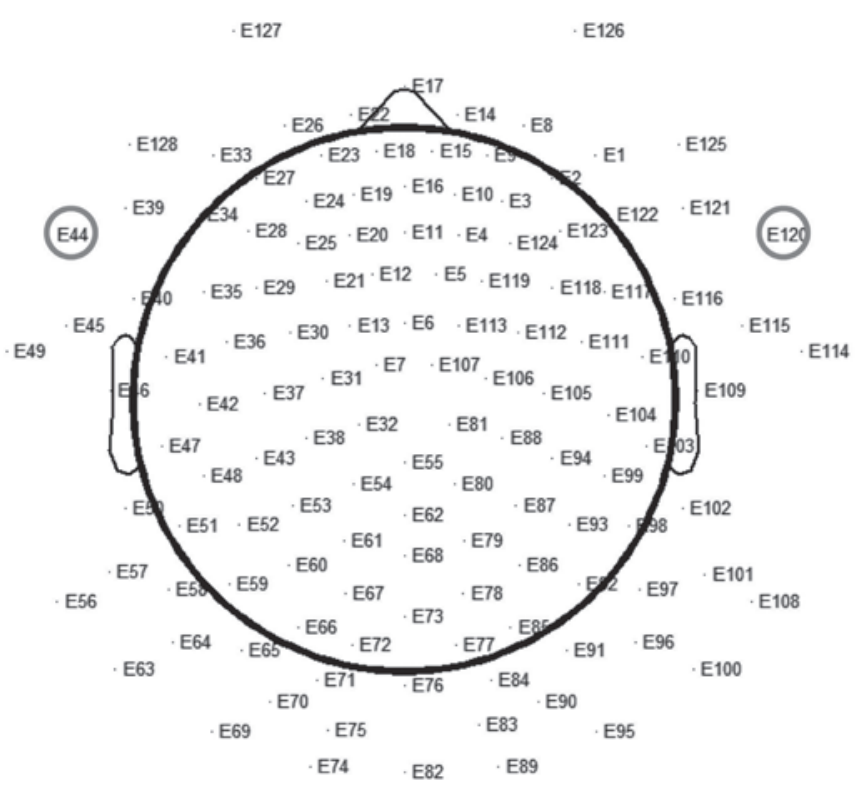

Figure 1. Location of the 128 electrodes according to the international 10-20 standard system. E44 and E120 postions are circled in gray.

them when they saw an upward arrow or a downward arrow presented on the computer screen. For passive movement with no MI task, subjects were asked not to move or imagine movement. The subjects were not allowed to see the instructions and the examiner moved the subject's figures according to the instructions on the screen. The subjects looked at the fixation cross on a nearby screen. For passive movement with MI, subjects were asked to imagine the same movements without actually performing them when they saw the arrows on the screen. The examiner moved the subject's figures according to the instructions on the screen. Each trial started with a $2 \mathrm{sec}$ presentation of a fixation cross in the center of the screen and the subjects began to perform the tasks when they saw the arrow stimulus continuously presented for $1 \mathrm{sec}$ on the screen. The inter-trial interval was $10 \mathrm{sec}$. Task switching instructions were presented on the computer screen for $2 \mathrm{~min}$. During this resting period, the subjects were asked to stay motionless and relax. An electromyogram (EMG) was recorded by a dense array 128 channel EEG system 200 (Electrical Geodesics Inc., Eugene, OR, USA) throughout the experiment to ensure that subjects did not move their hand during imagery of the movement.

In a 30 min training period, subjects familiarized themselves with the stimulus and practiced the timing of the passive movements and imaging movements. Subjects were trained to perform imagination without introducing muscular contraction and were reminded to use kinaesthetic imagery rather than visual imagery during the training and experimental period. The training length was a minimum of $30 \mathrm{~min}$ and was prolonged until the subjects were comfortable with the imagination task. EMG was recorded throughout the training period and the subjects were asked to practice imagining the movement until they were able to imagine the movement without an EMG response.

ERP measurement. The EEG was recorded by a dense array 128 channels EEG system (Electrical Geodesics Inc., Eugene, 


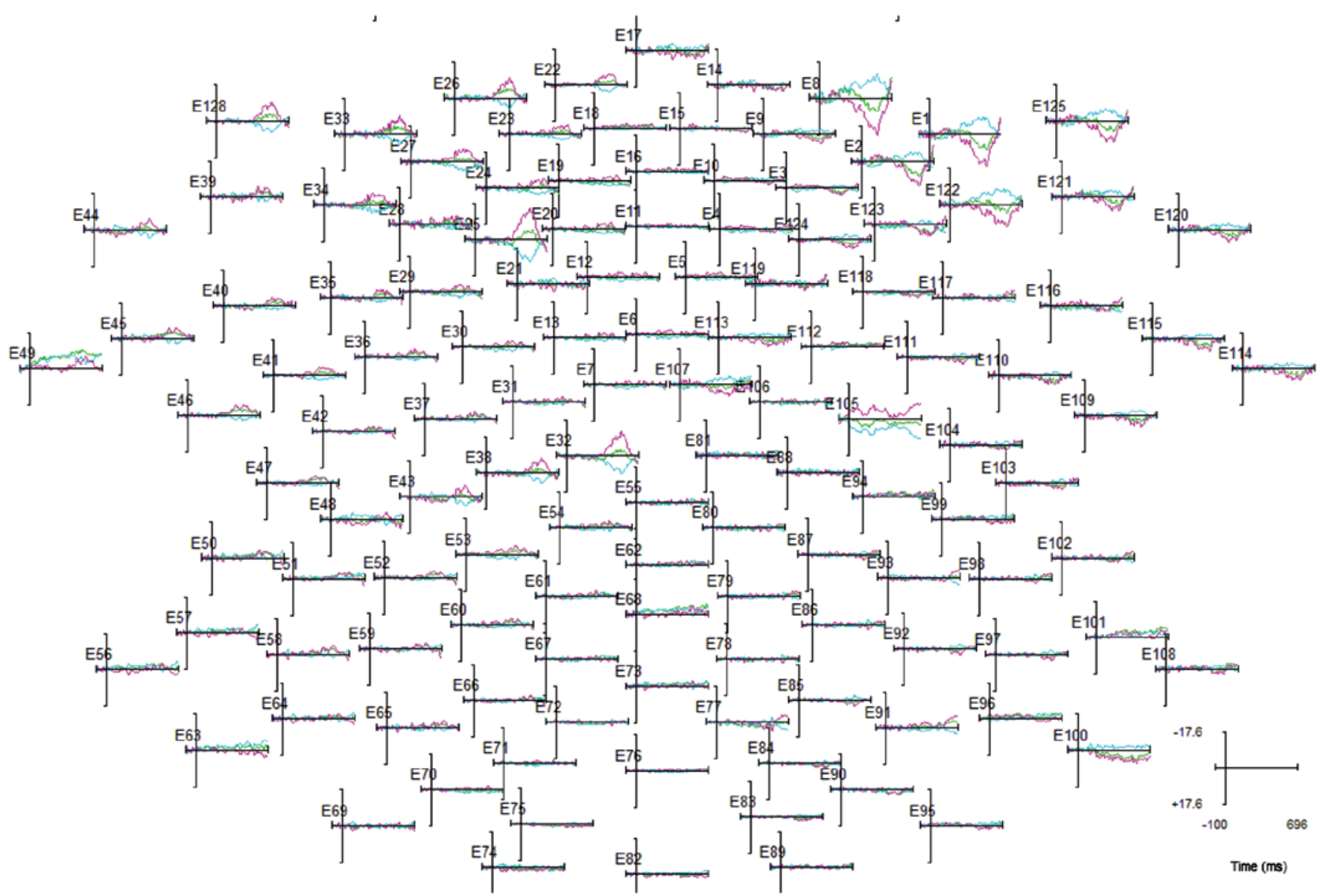

Figure 2. Averaged event-related potentials (ERPs) evoked by motor imagery in controls at 128 electrode sites over the scalp surface. The green and blue lines represent ERPs evoked by ipsilateral and contralateral finger movements, respectively. Red lines represent the difference between ERPs determined by subtracting the ERP amplitude of contralateral from ipsilateral finger or imaged movements.

OR, USA 200). The data were recorded from 128 scalp electrodes mounted on an elastic cap (Brain Products, Gilching, Germany) according to the international 10-20 system (Fig. 1). Vertical and horizontal electrooculograms were recorded separately. All electrodes were referenced to the mastoid surface during recording. The electrode impedance was kept below $5 \mathrm{k} \Omega$. Data were recorded continuously with a band pass of $0.05-40 \mathrm{~Hz}$ and a sampling rate of $500 \mathrm{~Hz}$ and stored on a hard disk for off-line analysis. The EEG recordings were analyzed according to the stimulus (MI, passive movement with no MI and passive movement with MI) and the sides of finger movement (left vs. right), and were averaged in each group separately. The EEG data were averaged for $800 \mathrm{~ms}$, including $100 \mathrm{~ms}$ prior to the onset of stimulus for baseline correction. The artifacts due to eye movement and blinking were removed from EEG recordings, using independent component analysis (25). Individual trials with excessive muscle activity ( $>100 \mu \mathrm{V}$ peak-to-peak amplitude) were excluded. The averaged data were re-referenced to an average voltage value across all electrodes.

Statistical analysis. Analyses were performed using SPSS 13.0 (SPSS, Inc., Chicago, IL, USA). All values are presented as the mean $\pm \mathrm{SD}$. One-way analysis of variance was used for comparisons. $\mathrm{P}<0.05$ was considered to indicate a statistically significant difference.

\section{Results}

Fig. 2 shows averaged ERPs evoked by MI in controls at 128 electrode sites over the scalp surface. The amplitude of ERPs evoked by imagined movement was distributed maximally over frontal scalp sites. As the inferior precentral area has been observed to be associated with the MI induced by the finger movement task (26), ERP data was selected and analyzed from two electrodes E44 and E120 that were positioned close to F9 and F10 (corresponding to the inferior precentral area) according to the international $10-20$ system.

For the controls, the lateralization effect was observed for ERPs evoked by passive movement with MI (Fig. 3A and B, and Fig. 4). The difference of ERPs was calculated by subtracting the ERP amplitude of contralateral from the ipsilateral finger or imaged movements. At the left electrode E44 (Fig. 3A), the difference between ERPs was negative during the 0-300 and positive during the 300-700 $\mathrm{ms}$ interval. By contrast, at the E120 right electrode (Fig. 3B), the difference between the ERPs was positive during the 0-300 and negative during the 300-700 ms interval. A similar effect, but to a lesser extent, was observed for ERPs evoked by MI alone (Fig. 3C and D). However, no lateralization effect was observed for ERPs evoked by passive movement with no MI (Fig. 3E and F). The difference between ERPs at 200 and $500 \mathrm{~ms}$ were significantly larger in patients with passive 

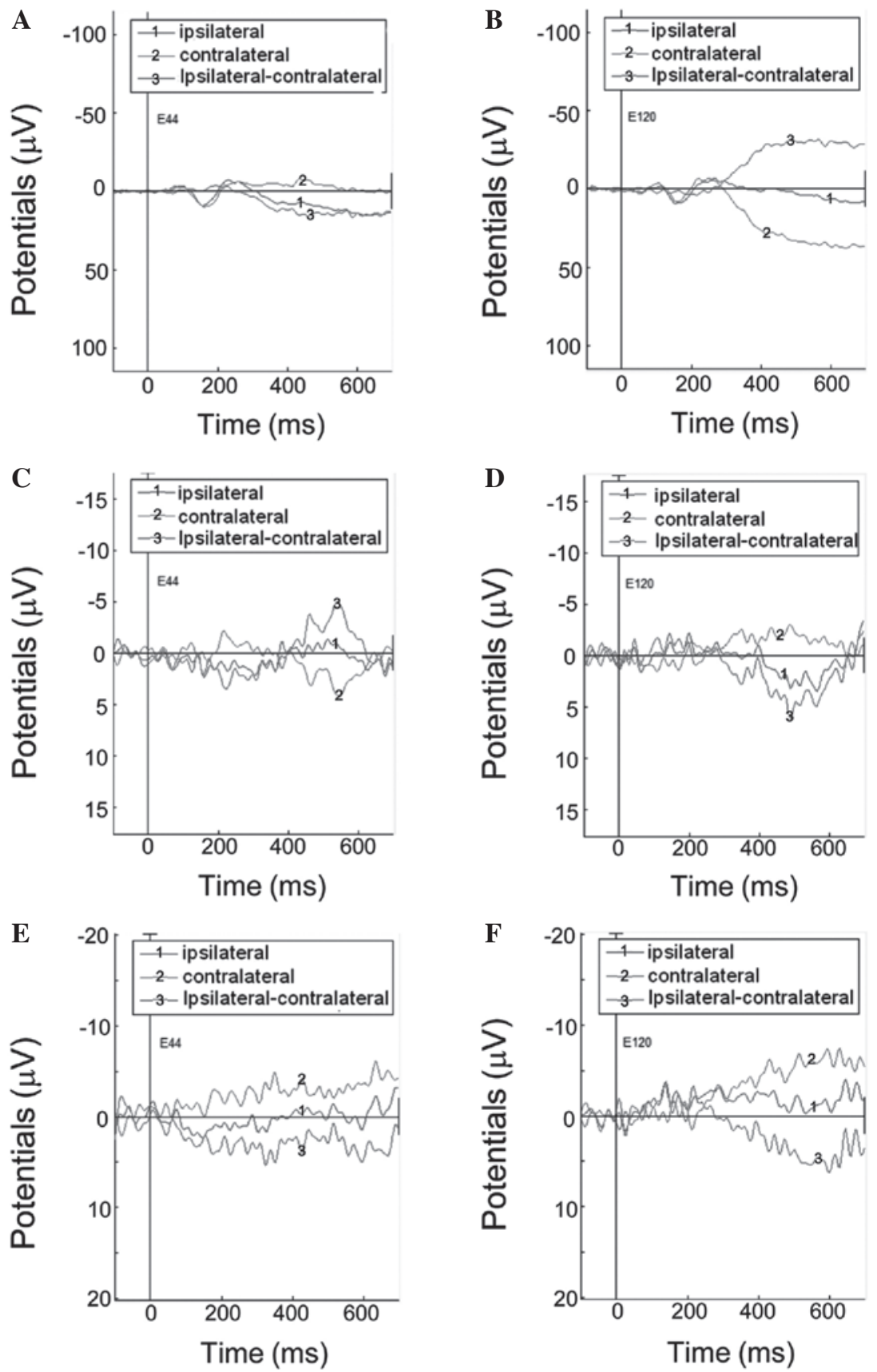

Figure 3. Average event-related potential (ERP) waveforms recorded over electrodes (A, C and D) E44 and (B, D and F) E120 in controls. ERPs were evoked by (A and B) passive movement with motor imagery; (C and D) imagined movement and; (E and F) passive movement with no motor imagery. The 1 and 2 lines represent ERPs evoked by ipsilateral and contralateral finger movements, respectively. Lines labeled with 3 represent the difference between ERPs determined by subtracting the ERP amplitude of contralateral from ipsilateral finger or imaged movements.

movement with MI than those with MI alone and with no MI $(\mathrm{P}<0.05$, Fig. 4A and B). The lateralization was also calculated by a ratio of the potential at $500 \mathrm{~ms}$ at electrode E120 to that at electrode E44 (Fig. 4C). A lateralization effect (negative lateralization ratio) was observed for ERPs evoked by passive movement with MI and MI alone, but not by passive movement without MI (Fig. 4C). The lateralization ratio in passive movement without MI was significantly different from that in passive movement with $\mathrm{MI}$ and $\mathrm{MI}$ alone $(\mathrm{P}<0.05)$.

For stroke patients, no lateralization effect was observed for ERPs evoked by passive movement with MI, imagined movement and passive movement with no MI (Figs. 4 and 5).
For passive movement with MI and MI alone, the difference between ERPs was negative during the 0-700 ms interval at the E44 and E120 electrodes (Fig. 5A-D). For passive movement without MI, the difference between ERPs were positive during the $0-700 \mathrm{~ms}$ interval at electrode E44, but negative at the electrode E120 (Fig. 5E and F). The amplitudes of the difference between ERPs at 200 and $500 \mathrm{~ms}$ were significantly smaller in stroke patients compared with those in the controls $(\mathrm{P}<0.05$, Fig. $4 \mathrm{~A}$ and $\mathrm{B})$. By contrast, the lateralization ratio in the stroke patients was opposite and significantly different from that of the controls $(\mathrm{P}<0.05$, Fig. 4C). 
A

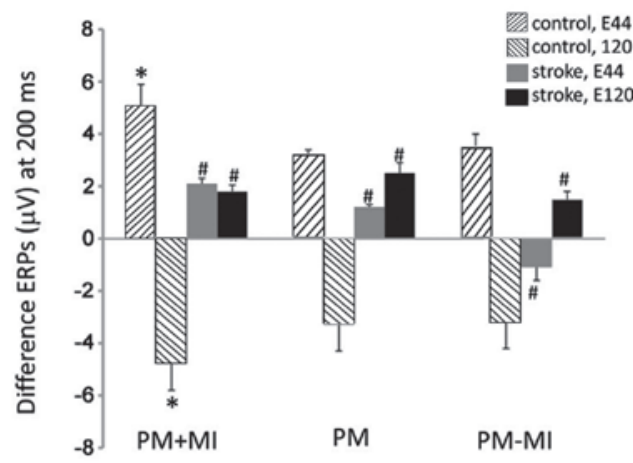

B

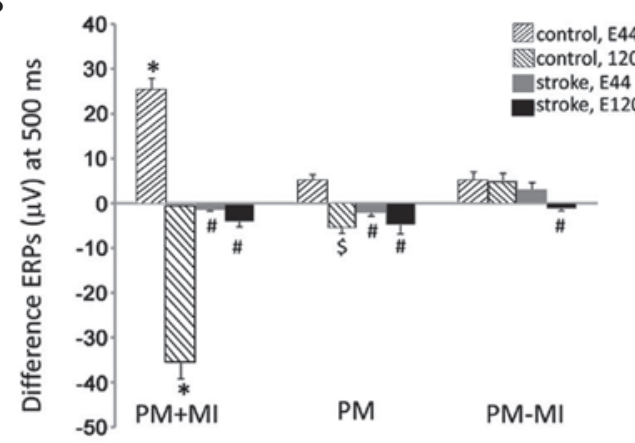

C

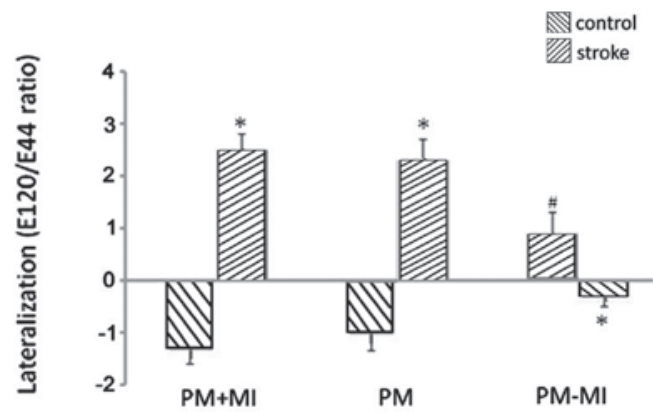

Figure 4. Lateralization effects for event-related potentials (ERPs) evoked by passive movement (PM) with motor imagery (MI), imagined movement and PM with no MI (PM-MI) in controls and stroke patients. (A and B) Difference between ERPs at (A) $200 \mathrm{~ms}$ and (B) $500 \mathrm{~ms}$ in controls (n=9) and stroke patients $(n=9) .{ }^{*} \mathrm{P}<0.05$ vs. $\mathrm{MI}$ and $\mathrm{PM}-\mathrm{MI}$ in controls; ${ }^{*} \mathrm{P}<0.05$ vs. controls and ${ }^{\$} \mathrm{P}<0.05$ vs. PM-MI in controls. (C) Lateralization ratios of ERPs in controls and stroke patients. Lateralization was calculated by determining the ratio of the potential at $500 \mathrm{~ms}$ at electrode E120 to that at electrode E44 ${ }^{*} \mathrm{P}<0.05$ vs. control and ${ }^{\#} \mathrm{P}<0.05$ vs. $\mathrm{PM}+\mathrm{MI}$ and $\mathrm{MI}$ in controls.

\section{Discussion}

The present study aimed to investigate the characteristic features in ERPs evoked by MI, passive movement without MI and passive movement with MI in healthy controls and stroke patients. Several predominant results were observed in ERPs which may be used to distinguish movement-related electrical activity of the brain between controls and stroke patients. The movement-related potentials were observed to be distributed maximally over the frontal cortex. The amplitudes of ERPs in stroke patients were smaller than those in the controls and for controls, the difference between ERPs exhibited different directions between the 0-300 and the 300-700 ms interval. However, this feature was absent in stroke patients. Furthermore, ERPs evoked by MI and passive movement with
MI exhibited lateralization effects in controls, but not in stroke patients. This lateralization effect was specific to MI as ERPs evoked by passive movement without MI did not exhibit the lateralization effect.

It is generally believed that MI and motor execution share a common neural substrate, including motor and premotor cortex (3). Concurrent with this hypothesis, it was identified that MI produces lateralization effects similar to passive movement with MI in the controls. The MI-induced lateralization effect was smaller compared with that evoked by passive movement with MI. This is concurrent with several studies that demonstrated that MI induced changes in brain activity are less pronounced than those induced by motor execution $(27,28)$. Furthermore, the lateralization effect is unique to MI as passive movement does not evoke it. Passive movement, similar to MI and motor execution, has been demonstrated to induce a lateralization effect in ERD with a stronger ERD contralateral to the movement $(22,23,29)$, suggesting that ERD is not a useful measurement to distinguish MI from passive movement. However, the lateralization effect in ERPs identified in the present study was unique to MI and therefore, may be used as a measurement to distinguish MI and passive movement.

The present study also demonstrated that the lateralization effect induced by MI or passive movement with MI observed in controls is not present in stroke patients, suggesting that MI-induced lateralization of brain activity is damaged in stroke patients. It has been suggested that the primary motor cortex (M1) on the side of the stroke affects lateralization during MI $(30,31)$. However, the lateralization effect was not identified in the inferior precentral area of either side of the brains of the stroke patients. This difference may be due to several reasons. Different studies include distinct patient populations; in the present study only patients with a disease duration of 3-6 months were selected, while two other studies $(30,31)$ included stroke patients with a disease duration of $\geq 8$ months. In addition, different regions of interest are investigated among studies. It has been suggested that in normal healthy subjects, the inferior precentral area is particularly associated with MI and the primary sensory and motor areas and anterior cerebellum are associated with marginal imagery activity (26). In the present study therefore, the inferior precentral area was selected, while other studies investigated the primary motor cortex (M1) $(30,31)$. Furthermore, previous functional MRI studies in chronic stroke patients showed that virtual reality practice only increased lateralization in the primary sensorimotor cortex, and not in other brain areas, such as the primary motor cortex (M1), premotor cortex, supplementary motor area and primary sensory cortex (32). Whether virtual reality practice or other interventions improve the MI-induced lateralization in ERPs in the inferior precentral area requires further investigation.

There are certain limitations to this study. As with all studies of MI, the present study did not confirm that participants actually performed the MI tasks as instructed. However, the tasks were very simple and all participants were instructed to tell the examiner of any difficulty in performing the MI tasks. In addition, the current study did not test ERPs induced by motor execution in these patients as the purpose of this study was to 
A
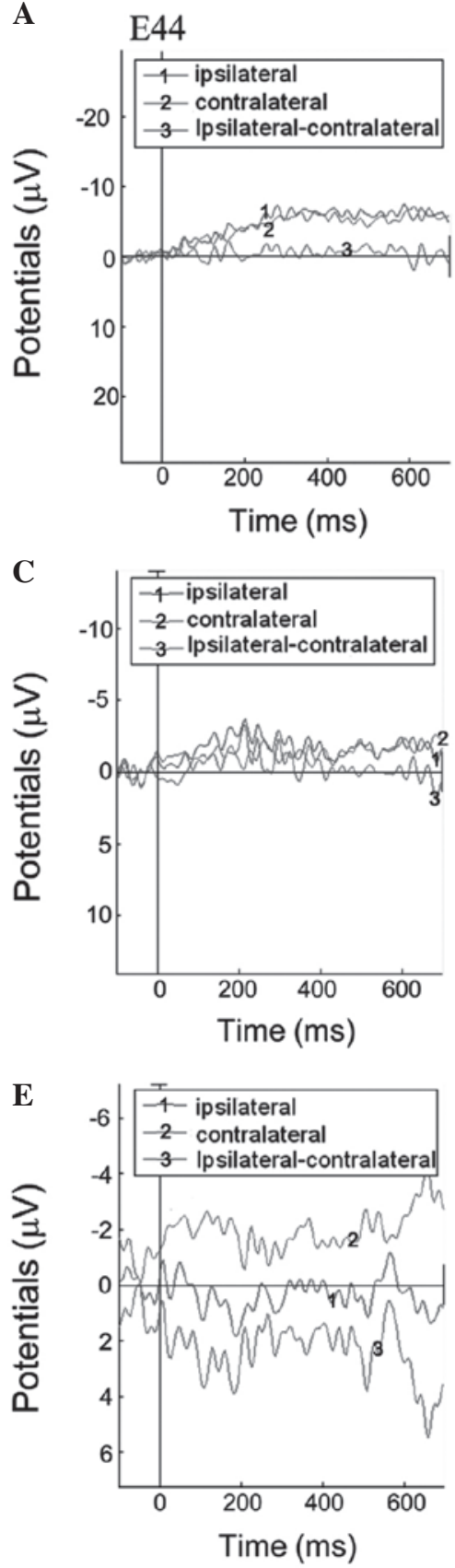

B
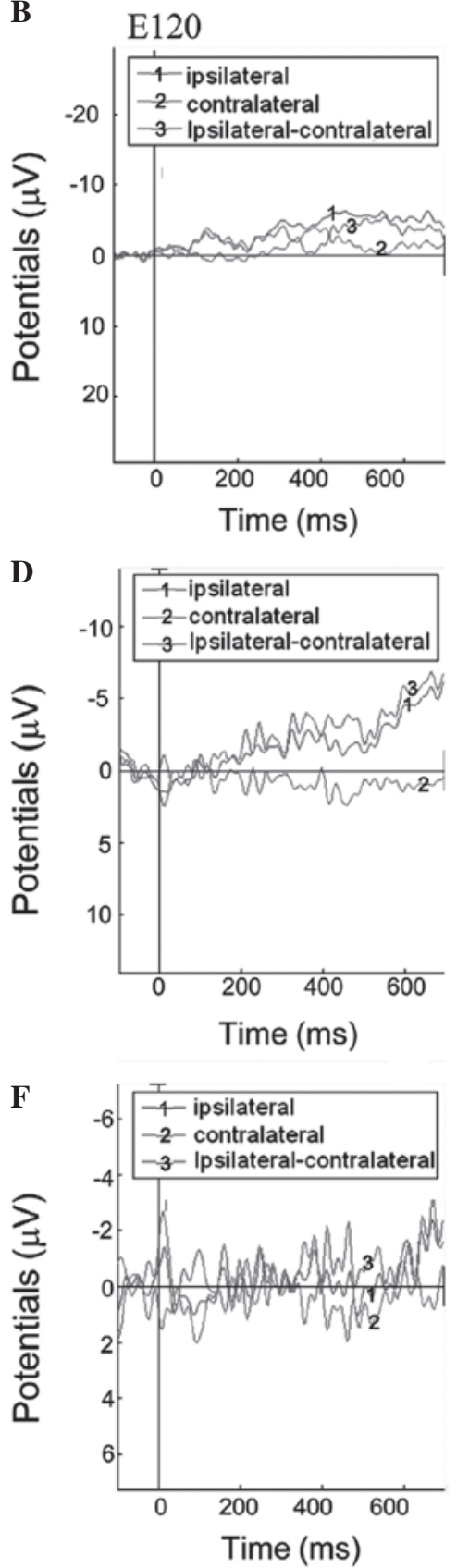

Figure 5. Average event-related potential (ERP) waveforms recorded over electrodes (A, C and D) E44 and (B, D and F) E120 in stroke patients. ERPs were evoked by (A and B) passive movement with motor imagery; (C and D) imagined movement and; (E and F) passive movement with no motor imagery. The 1 and 2 lines represent ERPs evoked by ipsilateral and contralateral finger or imaged movements, respectively. Lines labeled with 3 represent the difference between ERPs determined by subtracting the ERP amplitude of contralateral from ipsilateral finger or imaged movements.

identify the characteristic feature of ERPs induced by MI in the stroke patients, using a simple index finger movement task. Our future study concerning the difference in ERPs induced by MI and motor execution in stoke patients is under investigation, using various complex movement tasks. Moreover, only nine stroke patients are included in the present study. Thus, a study with a larger number of participants is required.

The present results implicate the use of MI in stroke rehabilitation. Several studies have shown that MI practice improves motor function in stroke patients (4-6). However, failure to measure MI ability is a predominant limitation to evaluate MI impairment and recovery following MI training in stroke patients. The MI-induced lateralization in ERPs observed in this study may be a useful measure to evaluate the MI in stroke patients. Future studies are required to determine whether the MI-induced lateralization in ERPs are recovered in these stroke patients following MI training.

In conclusion, MI-induced lateralization in stroke patients and healthy age-matched controls was investigated. It was demonstrated that MI-induced lateralization observed in healthy controls was not present in stroke patients. The results suggest that the MI-induced lateralization effect in ERPs may be a useful measure for evaluating MI impairment and recovery in stroke patients. 


\section{References}

1. Wade D: Rehabilitation therapy after stroke. Lancet 354: 176-177, 1999.

2. Braun SM, Beurskens AJ, Borm PJ, Schack T and Wade DT: The effects of mental practice in stroke rehabilitation: a systematic review. Arch Phys Med Rehabil 87: 842-852, 2006.

3. de Vries S and Mulder T: Motor imagery and stroke rehabilitation: a critical discussion. J Rehabil Med 39: 5-13, 2007.

4. Guttman A, Burstin A, Brown R, Bril S and Dickstein R: Motor imagery practice for improving sit to stand and reaching to grasp in individuals with poststroke hemiparesis. Top Stroke Rehabil 19: 306-319, 2012.

5. Page SJ, Levine P and Leonard AC: Effects of mental practice on affected limb use and function in chronic stroke. Arch Phys Med Rehabil 86: 399-402, 2005.

6. Dijkerman HC, Ietswaart M, Johnston M and MacWalter RS: Does motor imagery training improve hand function in chronic stroke patients? A pilot study. Clin Rehabil 18: 538-549, 2004.

7. Guillot A and Collet C: Contribution from neurophysiological and psychological methods to the study of motor imagery. Brain Res Brain Res Rev 50: 387-397, 2005.

8. Grush R: The emulation theory of representation: motor control, imagery, and perception. Behav Brain Sci 27: 377-442, 2004

9. Annett J: Motor imagery: perception or action? Neuropsychologia 33: 1395-1417, 1995.

10. Jeannerod M and Decety J: Mental motor imagery: a window into the representational stages of action. Curr Opin Neurobiol 5 727-732, 1995

11. Decety J, Perani D, Jeannerod M, Bettinardi V, Tadary B, Woods R, Mazziotta JC and Fazio F: Mapping motor representations with positron emission tomography. Nature 371: 600-602, 1994.

12. Porro CA, Francescato MP, Cettolo V, Diamond ME, Baraldi P, Zuiani C, Bazzocchi M and di Prampero PE: Primary motor and sensory cortex activation during motor performance and motor imagery: a functional magnetic resonance imaging study. J Neurosci 16: 7688-7698, 1996.

13. Porro CA, Cettolo V, Francescato MP and Baraldi P: Ipsilateral involvement of primary motor cortex during motor imagery. Eur J Neurosci 12: 3059-3063, 2000.

14. Munzert J and Zentgraf K: Motor imagery and its implications for understanding the motor system. Prog Brain Res 174: 219-229, 2009.

15. Malouin F, Richards CL, Jackson PL, Dumas F and Doyon J: Brain activations during motor imagery of locomotor-related tasks: a PET study. Hum Brain Mapp 19: 47-62, 2003.

16. Dickstein R and Deutsch JE: Motor imagery in physical therapist practice. Phys Ther 87: 942-953, 2007.

17. Pfurtscheller G and Klimesch W: Functional topography during a visuoverbal judgment task studied with event-related desynchronization mapping. J Clin Neurophysiol 9: 120-131, 1992.

18. Pfurtscheller G and Neuper C: Motor imagery activates primary sensorimotor area in humans. Neurosci Lett 239: 65-68, 1997.
19. Prasad G, Herman P, Coyle D, McDonough S and Crosbie J: Applying a brain-computer interface to support motor imagery practice in people with stroke for upper limb recovery: a feasibility study. J Neuroeng Rehabil 7: 60, 2010.

20. Platz T, Kim IH, Pintschovius H, Winter T, Kieselbach A, Villringer K, Kurth R and Mauritz KH: Multimodal EEG analysis in man suggests impairment-specific changes in movement-related electric brain activity after stroke. Brain 123: 2475-2490, 2000

21. Stepień M, Conradi J, Waterstraat G, Hohlefeld FU, Curio G and Nikulin VV: Event-related desynchronization of sensorimotor EEG rhythms in hemiparetic patients with acute stroke. Neurosci Lett 488: 17-21, 2011.

22. Alegre M, Labarga A, Gurtubay IG, Iriarte J, Malanda A and Artieda J: Beta electroencephalograph changes during passive movements: sensory afferences contribute to beta event-related desynchronization in humans. Neurosci Lett 331: 29-32, 2002.

23. Müller GR, Neuper C, Rupp R, Keinrath C, Gerner HJ and Pfurtscheller G: Event-related beta EEG changes during wrist movements induced by functional electrical stimulation of forearm muscles in man. Neurosci Lett 340: 143-147, 2003.

24. No authors listed: Special report from the National Institute of Neurological Disorders and Stroke. Classification of cerebrovascular diseases III. Stroke 21: 637-676, 1990.

25. Jung TP, Makeig S, Humphries C, Lee TW, McKeown MJ, Iragui $\mathrm{V}$ and Sejnowski TJ: Removing electroencephalographic artifacts by blind source separation. Psychophysiology 37: $163-178,2000$

26. Hanakawa T, Immisch I, Toma K, Dimyan MA, Van Gelderen P and Hallett M: Functional properties of brain areas associated with motor execution and imagery. J Neurophysiol 89: 989-1002, 2003.

27. Beisteiner R, Höllinger P, Lindinger G, Lang W and Berthoz A: Mental representations of movements. Brain potentials associated with imagination of hand movements. Electroencephalogr Clin Neurophysiol 96: 183-193, 1995.

28. Lang W, Cheyne D, Höllinger P, Gerschlager W and Lindinger G: Electric and magnetic fields of the brain accompanying internal simulation of movement. Brain Res Cogn Brain Res 3: 125-129, 1996.

29. Kaiser V, Kreilinger A, Müller-Putz GR and Neuper C: First steps toward a motor imagery based stroke BCI: new strategy to set up a classifier. Front Neurosci 5: 86, 2011.

30. Stinear CM, Fleming MK, Barber PA and Byblow WD: Lateralization of motor imagery following stroke. Clin Neurophysiol 118: 1794-1801, 2007.

31. Sabaté M, González B and Rodríguez M: Brain lateralization of motor imagery: motor planning asymmetry as a cause of movement lateralization. Neuropsychologia 42: 1041-1049, 2004.

32. You SH, Jang SH, Kim YH, Hallett M, Ahn SH, Kwon YH, Kim JH and Lee MY: Virtual reality-induced cortical reorganization and associated locomotor recovery in chronic stroke: an experimenter-blind randomized study. Stroke 36: 1166-1171, 2005. 